\title{
Regadenoson stress induced wall motion abnormalities during cardiac MRI
}

\author{
Kalindi Parikh*, Patricia W Bandettini, Marcus Y Chen, Jeannie H Yu, Sujata M Shanbhag, Andrew E Arai \\ From 18th Annual SCMR Scientific Sessions \\ Nice, France. 4-7 February 2015
}

\section{Background}

Wall motion abnormalities are central to dobutamine stress CMR but have not been studied with regadenoson. This study was designed to compare the diagnostic performance of regadenoson regional wall motion abnormalities (RWMA) versus first-pass perfusion in the detection of significant coronary artery disease (CAD).

\section{Methods}

Patients underwent regadenoson CMR that included 3 (basal, mid, apical) slices of rest and peak stress real-time cines, with matching stress and rest first-pass perfusion, and late gadolinium enhancement (LGE) imaging.

The reference standard for presence or absence of CAD was derived from invasive coronary angiography or coronary CT angiography (CTA). Invasive angiography established significant CAD based on a threshold of $\geq 70 \%$ stenosis and could rule-in or exclude CAD. CTA was only used to exclude CAD if the calcium score was $<100$ and no stenosis was $>30 \%$; CT was not used to diagnose CAD.

Two blinded, readers qualitatively scored RWM of pre and post-regadenoson.

\section{Results}

26 of 49 patients had at least one $70 \%$ coronary artery stenosis. The sensitivity and specificity of regadenoson perfusion for detecting significant CAD was 92 and 91\%, respectively. In comparison, the sensitivity and specificity of RWMA on stress imaging was 58 and $96 \%$, respectively, and a new or worsening RWMA on stress compared to rest yielded a sensitivity and specificity of 38 and $100 \%$, respectively. The sensitivity and specificity of LGE was 65 and 87\%, respectively. Inter-reader agreement for RWMA was good (kappa 0.63).
Table 1 Comparison of diagnostic accuracy of differing stress CMR exam components

\begin{tabular}{cccccc}
\hline & Sensitivity & Specificity & Accuracy & PPV & NPV \\
\hline Perfusion & 0.92 & 0.91 & 0.92 & 0.92 & 0.91 \\
\hline Stress RWMA & 0.58 & 0.96 & 0.76 & 0.94 & 0.67 \\
\hline Induced RWMA & 0.42 & 1.00 & 0.69 & 1.00 & 0.61 \\
\hline LGE & 0.65 & 0.87 & 0.76 & 0.85 & 0.69 \\
\hline
\end{tabular}

$\mathrm{PPV}=$ positive predictive value; $\mathrm{NPV}=$ negative predictive value; $\mathrm{RWMA}=$ regional wall motion abnormality; $L G E=$ late gadolinium enhancement

\section{Conclusions}

Although sensitivity is poor, a regadenoson-induced wall motion abnormality seen on CMR likely indicates significant CAD.

\section{Funding}

Funded by the Intramural Research Program of the National Heart, Lung, and Blood Institute (NHLBI), National Institutes of Health, Bethesda, MD.

Published: 3 February 2015

doi:10.1186/1532-429X-17-S1-P96

Cite this article as: Parikh et al:: Regadenoson stress induced wall motion abnormalities during cardiac MRI. Journal of Cardiovascular Magnetic Resonance 2015 17(Suppl 1):P96. 